\title{
Stability and composition of functional synergies for speech movements in children and adults
}

\author{
Hayo Terband ${ }^{1}$, Frits van Brenk ${ }^{1,2}$, Pascal van Lieshout ${ }^{3}$, \\ Lian Nijland ${ }^{1}$, Ben Maassen ${ }^{1,4}$ \\ ${ }^{1}$ Medical Psychology/Pediatric Neurology Centre/ENT, \\ Radboud University Nijmegen Medical Centre, Nijmegen, the Netherlands \\ ${ }^{2}$ Department of Speech and Language Therapy, University of Strathclyde, Glasgow, UK \\ ${ }^{3}$ Department of Speech-Language Pathology, Oral Dynamics Lab; Department of Psychology; \\ Institute of Biomaterials and Biomedical Engineering, University of Toronto, \\ and Toronto Rehabilitation Institute, Toronto, Canada \\ ${ }^{4}$ Department of Neurolinguistics, University of Groningen, Groningen, the Netherlands. \\ h.terband@mps.umcn.nl, frits.brenk@strath.ac.uk, p.vanlieshout@utoronto.ca, \\ 1.nijland@mps.umcn.nl, b.maassen@mps.umcn.nl
}

\begin{abstract}
The consistency and composition of functional synergies for speech movements were investigated in 7 year-old children and adults in a reiterated speech task using electromagnetic articulography (EMA). Results showed higher variability in children for tongue tip and jaw, but not for lower lip movement trajectories. Furthermore, the relative contribution to the oral closure of lower lip was smaller in children compared to adults, whereas in this respect no difference was found for tongue tip. These results support and extend findings of non-linearity in speech motor development and illustrate the importance of a multimeasures approach in studying speech motor development.

Index Terms: speech motor control, speech motor development, coordinative structures, speech movement patterns
\end{abstract}

\section{Introduction}

Speaking can be considered (one of) the most complex skills humans perform, and the development of speech motor control is a popular and well explored field of research. One way to study speech motor development is to focus on the consistency and stability of movement patterns. The development of speech motor skills essentially equates to the development of functional synergies of muscle activations or coordinative structures. In this way, the degrees of freedom are reduced, which makes the control task simpler. Consequently, as the speech production system matures the dynamic coordination among orofacial structures becomes more consistent $[1,2]$. The progression of speech motor development - i.e. the degree of functional synergy that reflects it - thus can be measured by assessing coordination and movement variability in speech production, a concept that has been successfully exploited in a fair number of studies.

Movement variability to some extent is expressed by the coefficients of variation of kinematic movement parameters (e.g. amplitude, duration and peak velocity). In addition, Smith and colleagues developed the spatiotemporal variability index (STI) as a method to examine the entire movement trajectory over time $[3,4,5]$. The STI captures the variability of multiple movement sequences whose trajectories are time and amplitude nor- malized. A lower STI value indicates a smaller deviation from the target movement template, and thus less variability.

Utilizing the STI, a recent longitudinal study has shown infants' articulator movements (jaw, upper lip, and lower lip) to become more stable over time and during linguistic/phonemic development [6]. Earlier group-studies yielded similar results, albeit not unambiguously. Measuring the words bob and pup, Smith and Goffman [7] found higher variability (STI) in lower lip movements of eight 4 year-olds as compared to a similar sized group of adults. Regarding 7 year-olds $(n=8)$ the results were not clear, that is the differences between the 7 year-olds and the adults did not reach statistical significance. In a second study (with the exact same set of subjects), Goffman and Smith [8] did find a significant difference in STI of lower lip movements among all three groups ( 4 year-olds $>7$ year-olds $>$ adults) in van, fan, ban, pan, and man. This study also investigated movement variability by means of the coefficients of variation of amplitude, peak velocity, and duration of lower lip movements, which results showed a similar pattern. Instead of focusing on a single articulator, Smith and Zelaznik [9] investigated the consistency of inter-articulator relationships (lip aperture and lower lip-jaw) in six groups of 30 children and adults ranging from 4 to 22 years of age. STI was measured over whole sentences. Results showed that also on the phrase-level variability decreases with age (albeit not linearly) and variability was found to be higher in 7 year-olds as compared to adults.

A second line of research investigates the development of functional synergies by focusing on the composition of functional synergies. The relative contribution of the different components that constitute speech movements undergoes large changes during development. Based on the prominent mandibular oscillations that are present from babbling onset, the jaw is thought to be predominant in early speech production [10]. Behavioral experiments investigating the development of jaw and lip coordination showed that infants (1- and 2 year-olds) rely largely on the jaw in the realization of oral closures, where 6-7 year-olds show a more adult-like differentiation between lip and jaw, which still undergoes refinement from there on [11, 12].

In the current study, we investigated both consistency and composition of functional synergies for speech movements in 
7 year-old children and adults in a reiterated speech task comprising the words spa (/spa:/) and paas (/pa:s/), which consist of the same sequence of speech sounds, but with a different syllabic structure (or in terms of Articulatory Phonology [13], of the same gestures but with different phasing). The stability of speech motor execution was assessed by calculating cyclic spatiotemporal variability index (cSTI) of the movement trajectories of jaw, lower lip and tongue tip. The cSTI is based on the STI and captures the variability of direction specific, cyclic movement patterns[14, 15]. Previous findings have shown a higher variability of lower lip movement trajectories for several age groups of children as compared to adults [7, 8]. Based on these results, we predict the variability of jaw and tongue tip movement trajectories also to be higher in 7 year-olds as compared to adults. The composition of synergies was assessed by the amplitudes of the movement components of tongue tip and lower lip in the realization of the constrictions for respectively the $/ \mathrm{s} /$ and the $/ \mathrm{p} /$. Additionally, we investigated the amplitude of jaw opening during the /a:/. As the relative contribution of the lower lip to oral closures has been shown to be smaller for children compared to adults $[11,12]$, we expect to find a similar smaller amplitude component for the tongue tip.

\section{Method and materials}

\subsection{Participants and data collection}

Six children ( 3 females and 3 males, 6;4-9;8 yrs;mnths, mean 7.5 SD 1.2) and eight adults (6 females and 2 males, $21 ; 3-27 ; 2$ yrs;mnths, mean 23.7 SD 2.2) participated in the study. All participants displayed speech, language, oro-motor and auditory abilities within the normal range. The children's data were collected as part of a study by Nijland [16], in which they participated as a control group. Two stimuli spa and paas were recorded in $5 \mathrm{sec}$. trials. The data of the adults were collected as part of another study [17], and were recorded in $12 \mathrm{sec}$. trials. The participants' task was to repeat the stimulus at a self-chosen normal, comfortable pace.

\subsection{Procedures}

Experimental procedures followed the same protocol as used in previous studies from our labs $[18,14,15]$. Articulatory data were collected using an AG100 Carstens Electro-Magnetic Midsaggittal Articulograph (EMMA) with time-aligned audio signal (Carstens Medizinelektronic, GmbH, Germany). Position data were sampled at $400 \mathrm{~Hz}$, acoustic data at $16 \mathrm{kHz}$. The transducer coils were attached in midline position to the upper and lower lip, jaw (lower incisors), tongue tip (0.5-1 $\mathrm{cm}$ behind the actual tongue tip), and tongue body ( $2 \mathrm{~cm}$ behind the tongue tip coil). In adults an additional coil was attached to the tongue dorsum ( $3 \mathrm{~cm}$ behind the tongue body coil). This coil was omitted in children due to a lack of space on the tongue, as well as for practical difficulties of attaching a coil to the tongue dorsum of children. Reference coils were placed on the nose bridge and on the gums of the upper incisors. All sensors were attached using surgical tissue glue (tongue \& gums; Henkel Indermil) or micropore sticky tape (lips \& nose). A helmet with three transmitter coils was placed on the participants' head. With this setup, it is possible to obtain 2-dimensional articulatory data in a biologically safe manner $[19,20]$.

After attaching the transducer coils and before the actual registrations, the participants were asked to answer a few questions about their daily pastime to enable them to familiarize themselves with the presence of the coils on their articulatory organs. Each sweep was preceded by a preparation interval in which the participants were told what to repeat. An orthographic description of the stimulus was visible on a computer screen during the trial. If necessary, the experimenter modeled the syllable once or twice. The acoustic speech sample was played back automatically over a connected speaker system after each trial during which the validity of the trial was judged by the experimenter. If any phonemic production errors, pauses, interruptions, or rate accelerations and decelerations occurred, the trial was repeated at the end of the series.

\subsection{Data processing}

Prior to analysis, raw articulatory data were corrected to compensate for variations in helmet positions, as well as rotational misalignments, i.e. 'twist' and 'tilt' movements of the head [21]. Subsequently, corrected data were processed in Matlab, following a procedure developed by Van Lieshout and colleagues $[14,15,20]$. Position data over time for all individual articulators were stored on a computer hard drive, separate for vertical (Y) and horizontal dimensions (X). The first part of the procedure consists of an algorithm for the assignment of peaks and valleys in the articulatory time series. In some cases where peaks and valleys were incorrectly defined by the algorithm, peak/valley assignment was corrected manually. The peak-topeak or valley-to-valley segment is referred to as a movement cycle (each cycle corresponds to a single utterance of the stimulus).

To calculate cSTI, individual direction-specific movement cycles (defined by the peaks and valleys in the signal) are amplitude- and time-normalized and aligned. At $2 \%$ intervals in relative time, separate standard deviations are then computed for the overlapping segments. The sum of these standard deviations within a plane of movement (vertical or horizontal) constitutes the cSTI [15].

Kinematic parameters (i.e. amplitude, velocity and duration; note that the current study only investigated amplitude) are derived from the position data for every movement cycle. In this case, lower lip and tongue tip signals were corrected for jaw movements using an estimate of jaw rotation based on the principal component of the mandible coil trajectory [14, 15, 22]. Thus, the lower lip and tongue tip kinematics reflect the unique contributions of these articulators for the production of $/ p /$ and /s/ respectively.

\subsection{Data analysis}

The number of cycles per trial differed per participant and per stimulus and was not equally distributed over the two groups. The children on average produced 6.3 consecutive cycles per trial, whereas the adults produced more than 10 consecutive cycles for every trial. Based on these numbers, the analyses were limited to a maximum number of 10 cycles per trial where available.

Separate analyses were performed for individual movement effectors (lower lip, tongue tip, and jaw). First, we used a repeated measures analysis of variance, with group (Children and Adults) as between-subject factor and task (paas and spa) as within-subjects factor. Since the results revealed no main or interaction effects for this factor, the data were collapsed over task and the final analysis of variance was performed with only group as between-subject factor. 


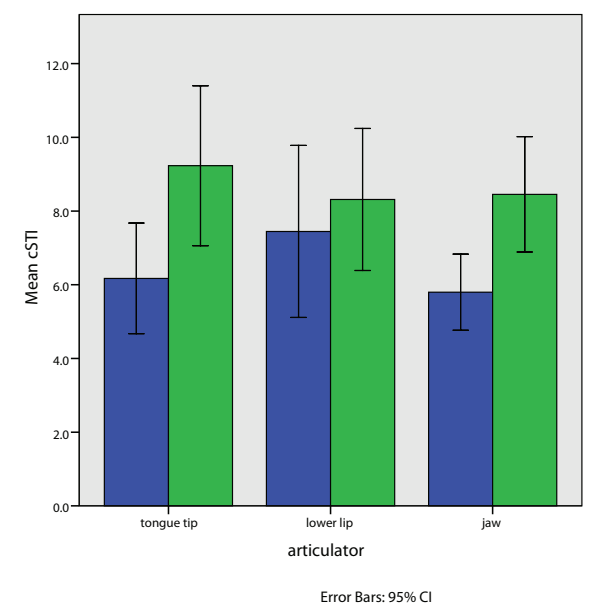

Figure 1: Mean variability of movement trajectories of tongue tip, lower lip, and jaw in the words /pa:s/ and /spa:/ in children vs. adults.

\section{Results}

Results on the variability of movement trajectories are presented in Figure 1. Statistical analysis revealed a higher variability for children compared to adults for tongue tip $[\mathrm{F}(1,12)=$ $6.740, \mathrm{p}<.05]$ and jaw $[\mathrm{F}(1,26)=10.219, \mathrm{p}<.01]$. No statistical difference was found with respect to movement trajectories of the lower lip.

Figure 2 presents the results on the composition of functional synergies for speech movements. The relative contribution of lower lip to the closure for the $/ \mathrm{p} /$ was shown to be smaller for children than for adults $[\mathrm{F}(1,26)=7.627, \mathrm{p}<$ $.01]$. Furthermore, the amplitude of jaw opening during the /a:/ proved to be larger in children than in adults $[\mathrm{F}(1,26)=5.034$, $\mathrm{p}<.05]$. No significant difference was observed for tongue tip in the realization of the constriction for the $/ \mathrm{s} /$.

\section{Discussion}

The purpose of this study was to investigate the consistency and composition of functional synergies for speech movements of children and adults. In summary, results showed a higher variability of jaw and tongue tip movement trajectories in 7 yearold children compared to adults. The children also exhibited a smaller relative contribution of lower lip and a larger amplitude of jaw opening movements. Overall, these results correspond to common conceptions about speech development. Additionally, results reveal interesting patterns across articulators.

Previous studies that investigated the variability of lower lip movement trajectories for 7 year-olds compared to adults yielded ambiguous results $[7,8]$. In these studies, variability was measured by the STI of words that were embedded in a sentence. In the present study, results revealed no statistical difference between a group of 6-9 year-old children children and adults in the variability of lower lip movement trajectories in a word-level repetitive speech task. Together with the ambiguity in previous findings, these results indicate that if there are differences in the stability of the lower lip-jaw synergy, they are subtle. Further insight is provided by investigating the composition of the lower lip-jaw synergy. Results showed the relative contribution of lower lip to the closure for the $/ \mathrm{p} /$ to be significantly

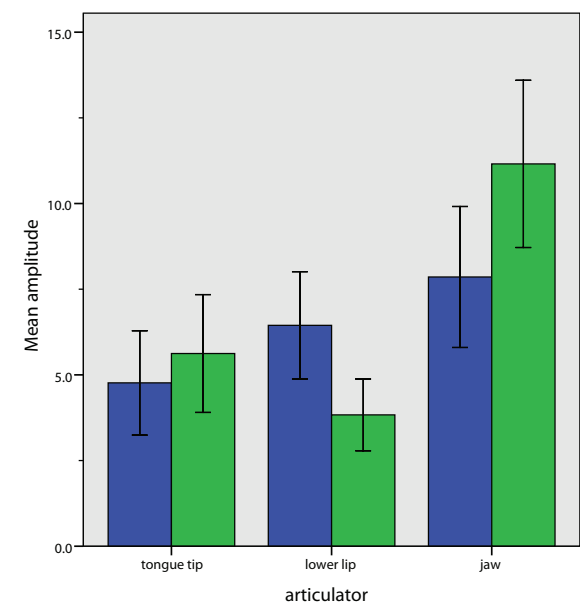

group DCults

Error Bars: 95\% CI

Figure 2: Relative contribution of articulators to functional synergies for speech movements in children vs. adults. Bars represent mean amplitudes of the movement components of tongue tip and lower lip in the realization of the constrictions for respectively the $/ \mathrm{s} /$ and the $/ \mathrm{p} /$, and the amplitude of jaw opening during the /a:/ in the words /pa:s/ and /spa:/.

smaller for children than for adults. Thus, whereas in terms of stability of the lower lip-jaw synergy at the level of individual movement cycles, speech motor development approaches adultlike qualities at the age of 7-8, a close inspection of kinematic variables shows that in fact children at this age may still differ from adults in the relative contribution of lower lip in bilabial closure gestures. These results confirm and extend earlier findings of Green and colleagues [11, 12]. Although children show a more adult-like differentiation between lip and jaw around the age of 7 , at this stage the lip-jaw coordinative structure still is not yet fully developed and still undergoes refinement.

The current study extends previous research on the development of speech movement coordination by also investigating movement trajectories of tongue tip. In this respect, results showed a pattern that is different from what we found for lower lip movements. As predicted, tongue tip movement trajectories exhibited a higher variability for children as compared to adults. Contrary to expectations, we found no difference in the relative contribution of tongue tip to the realization of the constriction for the $/ \mathrm{s} /$. However, without a reference of tongue-jaw contributions earlier in development the latter result is hard to interpret. Adult studies have shown the jaw's relative contribution to consonants to vary depending on phonological context [23] and manner of articulation [24]. Further research on tongue tip-jaw relations in younger children is needed.

\section{Conclusions}

The current study suggests that at the level of individual movement cycles, speech motor development follows different trajectories for different articulators. The stability of the lower lip-jaw functional synergy appears to have reached adult-like qualities at the age of 7-8, but a close inspection of kinematic variables shows that there are still differences in terms of the composition of the synergy. In lower lip-jaw, children relied to a large extent on closing the jaw in the realization of oral closures, where 
adults show a more clear contribution of the lower lip. These results are in line with the notion that the jaw is more dominant in children in the relative contribution to oral closures. Tongue tip, however, showed a different pattern. Results showed an adultlike composition of the tongue tip-jaw coordinative structure in 7 year-old children compared to adults, but with higher variability. However, without knowledge of the characterististics of the tongue tip-jaw functional synergy earlier in development, it is not possible to infer whether the developmental trajectory of the tongue tip-jaw synergy is qualitatively different from the lower lip-jaw synergy, or only shifted in time. Obtaining data of tongue tip movements in younger children constitutes a challenge for further research.

Overall, the present results support and extend findings of non-linearity in speech motor development. This illustrates that a true understanding of speech motor development requires the detailed investigation of multiple variables.

\section{Acknowledgements}

Authors would like to thank Sarah Klein and Aukje Lingsma for their help in data collection.

\section{References}

[1] Bernstein, N., "The coordination and regulation of movements", Oxford: Pergamon, 1967.

[2] Kelso, J.A., Tuller, B., Vatikiotis-Bateson, E., and Fowler, C.A., "Functionally specific articulatory cooperation following jaw perturbations during speech: evidence for coordinative structures", Journal of Experimental Psychology: Human Perception and Performance, 10(6):812-832, 1984.

[3] Smith, A., Goffman, L., Zelaznik, H.N., Ying, G., and McGillem, C., "Spatiotemporal stability and patterning of speech movement sequences", Experimental Brain Research, 104(3):493-501, 1995.

[4] Smith, A., Johnson, M., McGillem, C., and Goffman, L., "On the assessment of stability and patterning of speech movements", Journal of Speech, Language and Hearing Research, 43(1):277286, 2000.

[5] Lucero, J.C., "Comparison of measures of variability of speech movement trajectories using synthetic records", Journal of Speech, Language and Hearing Research, 48(2):336-344, 2005.

[6] Grigos, M.I., "Changes in articulator movement variability during phonemic development: a longitudinal study", Journal of Speech, Language and Hearing Research, 52(1):164-177, 2009.

[7] Smith, A. and Goffman, L., "Stability and patterning of speech movement sequences in children and adults", Journal of Speech, Language and Hearing Research, 41(1):18-30, 1998.

[8] Goffman, L. and Smith, A., "Development and phonetic differentiation of speech movement patterns", Journal of Experimental Psychology: Human Perception and Performance, 25(3):649660, 1999.

[9] Smith, A. and Zelaznik, H.N., "Development of functional synergies for speech motor coordination in childhood and adolescence", Developmental Psychobiology, 45(1):22-33, 2004.

[10] MacNeilage, P.F. and Davis, B.F., "Acquisition of speech production: Frames, then content", in M. Jeannerod [Ed], Attention and performance XIII: Motor representation and control, 453476, Hillsdale, NJ: Lawrence Erlbaum, 1990.

[11] Green, J.R., Moore, C.A., Higashikawa, M., and Steeve, R.W., "The physiologic development of speech motor control: lip and jaw coordination", Journal of Speech, Language and Hearing Research, 43(1):239-255, 2000.

[12] Green, J.R., Moore, C.A., and Reilly, K.J., "The sequential development of jaw and lip control for speech", Journal of Speech, Language and Hearing Research, 45(1):66-79, 2002.
[13] Browman, C.P. and Goldstein, L.M., "Articulatory phonology: an overview", Phonetica, 49(3-4):155-180, 1992.

[14] van Lieshout, P.H.H.M., Rutjens, C.A.W., and Spauwen, P.H.M., "The Dynamics of Interlip Coupling in Speakers With a Repaired Unilateral Cleft-Lip History", Journal of Speech, Language and Hearing Research, 45(1):5-19, 2002.

[15] van Lieshout, P.H.H.M., Bose, A., Square, P.A., Steele, C.M., "Speech motor control in fluent and dysfluent speech production of an individual with apraxia of speech and Broca's aphasia", Clinical Linguistics \& Phonetics, 21(3):159-188, 2007.

[16] Nijland, L., "Speech perception in children with speech output disorders", Clinical Linguistics \& Phonetics, 23(3):222-239, 2009.

[17] van Brenk, F., Terband, H., van Lieshout, P.H.H.M., Lowit, A., and Maassen, B., "An analysis of speech rate strategies in aging", Proceedings of the 10th Annual Conference of the International Speech Communication Association: Interspeech 2009, 6-10 September 2009, Brighton, UK.

[18] Nijland, L., Maassen, B., Hulstijn, W., and Peters, H.F.M., "Speech motor coordination in Dutch-speaking children with DAS studied with EMMA", Journal of Multilingual Communication Disorders, 2(1):50-60, 2004.

[19] Hasegawa-Johnson, M., "Electromagnetic exposure safety of the Carstens articulograph AG100", Journal of the Acoustical Society of America, 104(4):2529-2532, 1998.

[20] van Lieshout, P.H.H.M. and Moussa, W., "The assessment of speech motor behavior using electromagnetic articulography", The Phonetician, 1:9-22, 2000.

[21] Hoole, P. and Nguyen, N., "Electromagnetic articulography in coarticulation research", Forschungsberichte des Instituts fur Phonetik und Sprachliche Kommunikation der Universität München, 35:177-184, 1997.

[22] Westbury, J.R., Lindstrom, M.J., and McClean, M.D., "Tongues and Lips Without Jaws: A Comparison of Methods for Decoupling Speech Movements", Journal of Speech, Language and Hearing Research, 45(4):651-662, 2002.

[23] Hertrich, I. and Ackermann, A., "Lipjaw and tonguejaw coordination during rate-controlled syllable repetitions", Journal of the Acoustical Society of America, 107(4):2236-2247, 2000.

[24] Mooshammer, C., Hoole, P., and Geumann, A., "Jaw and Order", Language and Speech, 50(2):145-176, 2007. 